\title{
The Compression Method of Point Cloud Data based on the Hypotenuse-height Deviation Method
}

\author{
Wan Chenghui ${ }^{a}, \mathrm{He}$ Xiping ${ }^{\mathrm{b}}$ \\ School of Water Conservancy and Ecological Engineering, Nanchang Institute of Technology, \\ Nanchang 330099, Jiangxi Province, China \\ awww1994931@tom.com, ${ }^{\text {b387805465@QQ.com }}$
}

Keywords: point cloud compression, hierarchy, hypotenuse-height difference, linear feature, plan curvature

\begin{abstract}
The article describes a hypotenuse-height deviation compression method of magnanimous point cloud data based on hierarchy. Firstly the point cloud is stratified into several hierarchies which turn into linear point clouds, then compressed by using hypotenuse-height deviation threshold. By experiment, the compression effect of the method brought up is compared with that of several traditional point cloud compression methods, which verify the capability of realizing high-efficiency compression with abundant detailed features retained.
\end{abstract}

\section{Introduction}

Terrestrial 3D laser scanner is able to acquire the magnanimous ground point data quickly, while tens of millions of three-dimensional coordinates is blocking the post-processing, data transmitting and post data application, which reduces the computational efficiency and the application fields. Thus, the compression of the point cloud is needed ${ }^{[1]}$. The main point cloud compression algorithms can be classified into three ${ }^{[2]}$ : the compression based on point cloud ${ }^{[3]}$, the compression based on the grid topology relation ${ }^{[4][5]}$ and the compression based on surface fitting ${ }^{[6]}$. With an increasing number of point cloud data, the efficient and concise compression algorithms based on point cloud are already in the mainstream of the research of point cloud data compression algorithm. The compression algorithms based on point cloud includes: uniform grid sampling, curvature compression based on point method vector, extraction and compression of fairing features of projected planar point cloud based on hierarchy, compression of extracted features based on octree division, etc.

\section{Extraction of Point Cloud Data based on Hierarchy}

In order to compress the topographical point cloud data based on the planar curvature, a method of stratified extracting of point cloud data based on the point cloud hierarchy is applied. As shown in Figure 1, the scanned mountain point cloud is stratified along the elevation direction with a certain thickness, and the same-thickness point cloud data from each hierarchy is compressed and processed. The thickness $h$ stratified directly determines the number of point clouds projected in the grids of the upper and lower hierarchies, therefore the average value of height differences of point clouds between certain hierarchies is used as the reference.

$h=\beta \frac{1}{k} \sum_{i}^{k} h_{i}, i=1,2, \ldots, k$

Where $\beta$ represents the scaling factor of stratified thickness, $\mathrm{k}$ represents the number of point clouds between hierarchies and $h_{i}$ represents the height differences between point clouds. 
Fig.1 Stratifying Mode

\section{Point Cloud Compression Theory based on Hypotenuse-height Deviation Method}

This article adopts the hypotenuse-height deviation compression method, one of the point compression methods that leads to a good result for linear point cloud data.

The hypotenuse-height deviation method is data compression based on the hypotenuse-height differences, with two parameters for the choice of compressing points: the maximum hypotenuse-height difference and hypotenuse lengths between two chosen compressing points.As shown in Figure 2(a), two terminal points $p_{i-1}\left(x_{1}, y_{1}\right)$ and $p_{i+1}\left(x_{3}, y_{3}\right)$ are connected to form a base line. The distances from other points to the base line is calculated and the point $p_{i}\left(x_{2}, y_{2}\right)$ with the maximum distance herein is selected and recorded. The general equation of straight line is: $A x+B y+C=0$

By plugging in the coordinates of the two terminal points then we have:

$\left(y_{3}-y_{1}\right) x+\left(x_{1}-x_{3}\right)+\left(x_{3} y_{1}-x_{1} y_{3}\right)=0$

Where $A=y_{3}-y_{1}, B=x_{3}-x_{1}, C=x_{3} y_{1}-x_{1} y_{3}$.

Base on the distance equation from point to straight line we have:

$d=\frac{\left|A x_{2}+B y_{2}+C\right|}{\sqrt{A^{2}+B^{2}}}$

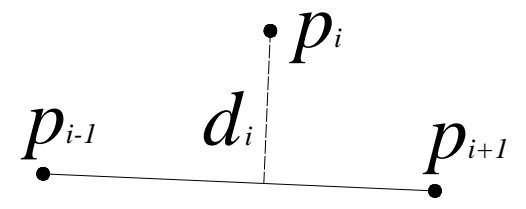

a) Terminal points and hypotenuse height

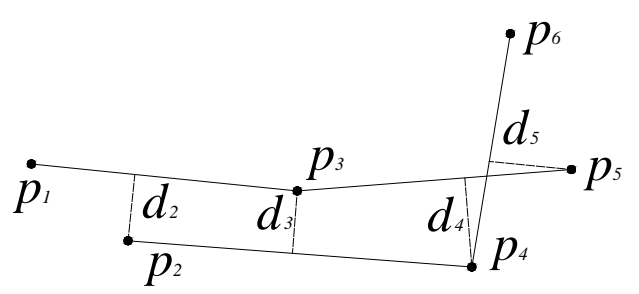

(b) Hypotenuse height threshold judgment

Fig. 2 The Theory of Hypotenuse-height Deviation Method

After the calculating of hypotenuse-height distance $d, p_{i-1}, p_{i}$ and $p_{i}, p_{i+1}$ are set as the terminal points of two straight lines, the maximum hypotenuse height is then selected and put into the chosen data set, and so it goes on. When the hypotenuse height threshold is selected, in a recursive division the data point between two points with hypotenuse height less than the threshold given, which means the curvatures variation between two data points is very tiny, will be therefore discarded. Owning to the relationship between hypotenuse height and curvature, the density of sampling points varies with relative curvature. A higher curvature usually results in denser sampling points.

\section{The Test and Analysis of Hypotenuse-height Deviation Method}

The algorithm is programmed on computer software Visual C++6.0, and the data used is shown in Figure3. The denoising mountain cloud data reaches 1.82 million.

Figure 3 indicates the comparison of the compressions of some point clouds in the 50th slice using different hypotenuse-height thresholds. Figure 3(a) shows the planar display of projected point cloud data from the $50^{\text {th }}$ slice with the cloud point number as 1,056.. Figure 3(b) with threshold at $0.25 \mathrm{~m}$ compression rate of approximately $70 \%$ is shown respectively. The profile features is basically retained in the hypotenuse-height compression in Figure 3(b) where the compression rate is higher to approximate $70 \%$. Therefore when choosing hypotenuse-height threshold value as $0.25 \mathrm{~m}$, stratified point cloud will be with better compression rate and features are well retained. 


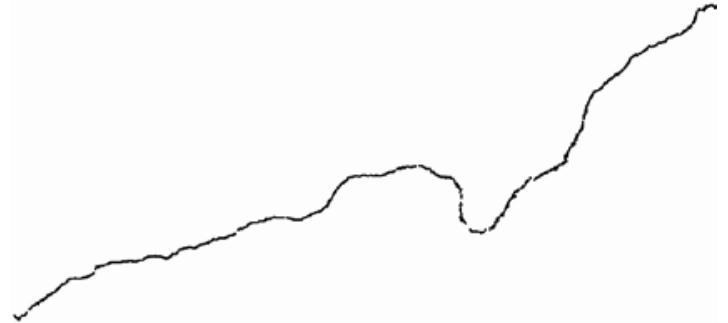

(a) The point cloud data in the $50^{\text {th }}$ slice $(n=1,056)$

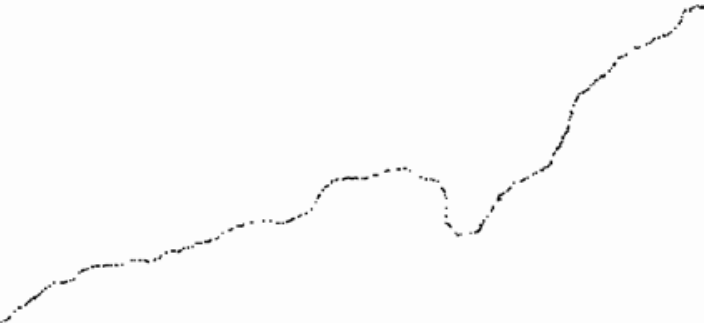

(b) Reference hypotenuse-height threshold as $0.25 \mathrm{~m}(\mathrm{n}=327)$

Fig. 3 Hypotenuse-height threshold compression of stratified point cloud

Uniform sampling, curvature sampling, grid bounding method and the hypotenuse-height deviation compression algorithm presented in this article, are compared for approximate $80 \%$ compression of mountain point cloud in Figure 4. In Figure 4(a), the uniform sampling results in unobvious compression of features. Compression using curvature sampling in Figure 4(b) shows good retaining of features but the inhomogeneous distribution of point clouds. The grid bounding method in Figure 4(c) results in unobvious retaining of curvature features. In Figure 4(d) ,the compression method based on hypotenuse-height deviation presented in this article, lead to obvious retaining of curvature features and homogeneous distribution of point clouds.

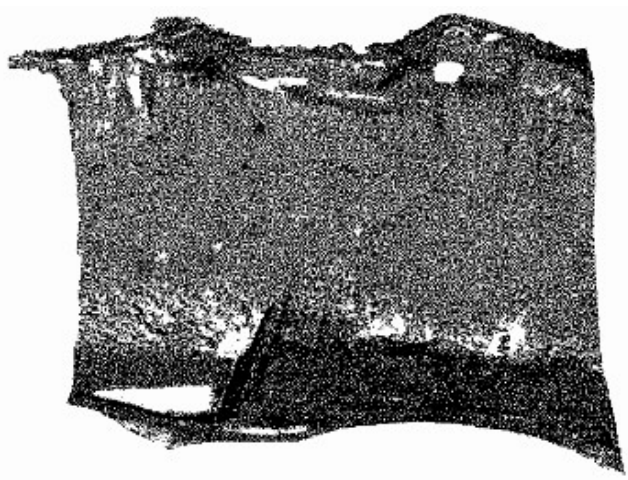

(a) Uniform sampling

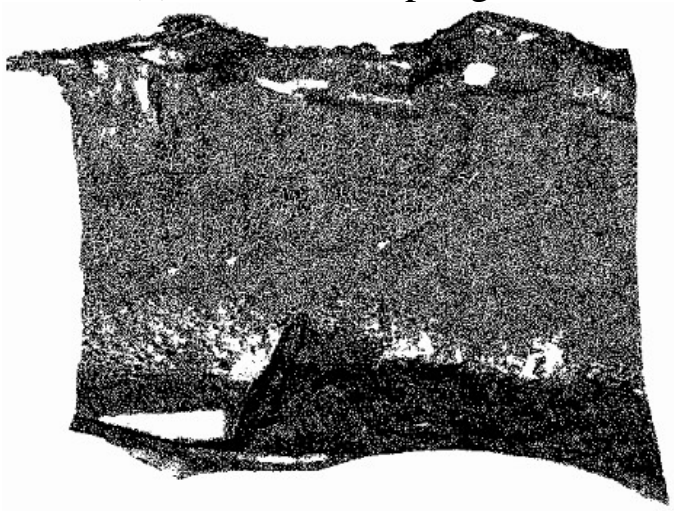

(c) Grid bounding method

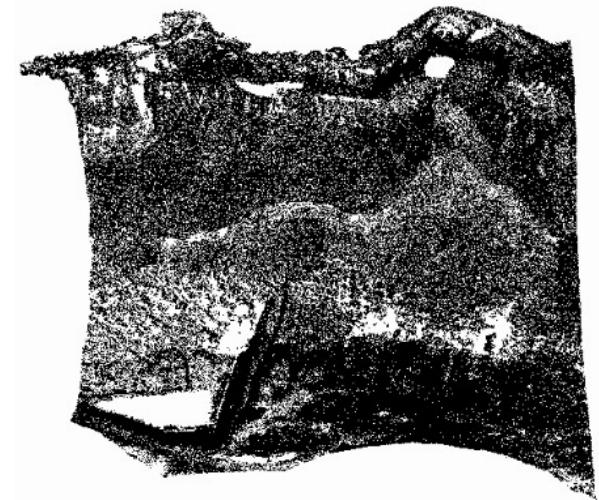

(b) Curvature sampling

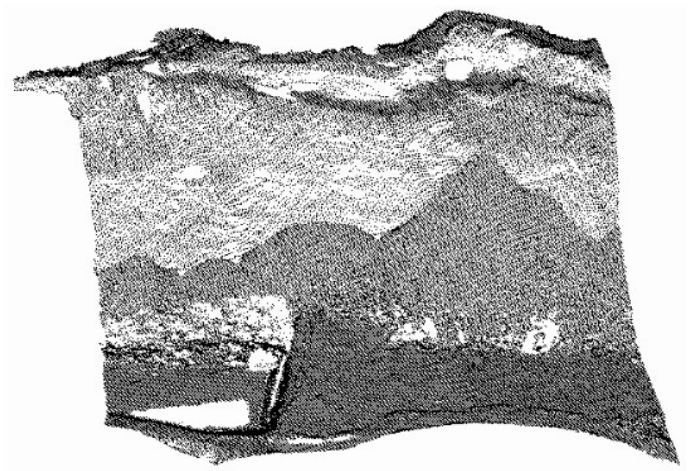

(d) The algorithm presented in this article

Fig.4. The result comparison of multiple compression methods

\section{Conclusions}

Hypotenuse-height deviation compression method based on point clouds, extracts point cloud by using stratifying, compresses the magnanimous point cloud data quickly. The difference from other compression method is that it realizes compressing adopting the hypotenuse-height threshold method after transforming the point clouds into two-dimension planer point clouds. The method realizes quick compressing using hierarchy strategy, and retained the curvature features of point cloud well with the application of hypotenuse-height threshold. This compression method can be 
used in certain practice.

\section{Acknowledgments}

Many thanks to the funding support of No. 20151BAB207051 project of science and technology office of Jiangxi province.

\section{References}

[1] Shi Y T. Research of key technology of 3D simulation of real tree based on point cloud [D]. Tongji University, 2012.

[2] Sun R, Zhang C X. Summary of the algorithms of point cloud data compression [J]. Technical Information, 2010, Vol.32: 253-255

[3] Zhang L Y. Research of reduction techniques of magnanimous measured data [J]. Journal of computer aided design and graphics. 2001, 13(11): 1019-1023.

[4] Lee K H, Woo H, Suk T. Point Data Reduction Using 3D grids [J]. The International Journal of Advanced Manufacturing Technology. 2001, 18: 201-210.

[5] Liu C, Wu H B. A data compression method of 3d laser scanning based on real 3d TIN [J]. Journal of Wuhan University (Science and technology edition). 2006(10): 908-911.

[6] Zhou L, Lin H, Zhong Y X. Research of the reduction of measured point cloud in surface reconstruction [J]. Informatization of Chinese Manufacturing Industry, 2004, Vol.33 (5): 102-105. 